\title{
Construcción de un Sistema para la Gestión de Proyectos con Empresas en una Carrera de Ingeniería
}

\author{
Rodolfo Schmal y Sabino Rivero \\ Escuela de Ingeniería en Informática Empresarial, Universidad de Talca, 2 Norte 685, Talca-Chile \\ (e-mail: rschmal@utalca.cl)
}

Recibido Dic. 14, 2015; Aceptado Feb. 18, 2016; Versión final Abr. 14, 2016, Publicado Ago. 2016

\begin{abstract}
Resumen
Se presenta un sistema de información que posibilita el seguimiento del estado de los proyectos ejecutados por los alumnos en empresas e instituciones y la emisión de informes. La Escuela de Ingeniería en Informática Empresarial de la Universidad de Talca, Chile, tiene un modelo de trabajo con empresas que ha sido valorado positivamente por alumnos, profesores y empleadores, al facilitar una inserción laboral temprana de sus egresados. El modelo fue formalizado mediante un diagrama de notación de modelamiento de procesos de negocios (BPMN). En él, todo proyecto es desarrollado por grupos de alumnos en una empresa y en el marco de un módulo de un curso. Esta formalización se ha visto complementada con el desarrollo del sistema de flujo de trabajo que se propone. Este permite registrar y monitorear información asociada a los proyectos que se desarrollan, las empresas en las que se realizan, los alumnos que los ejecutan, y los profesores y profesionales de la empresa involucrados.
\end{abstract}

Palabras clave: sistema de información; procesos; modelo de trabajo; universidad-empresa

\section{Building an Enterprise System for Management of Projects in a Career of Engineering}

\begin{abstract}
An information system that allows tracking the progress of projects executed by students $n$ companies and institutions and the preparation of reports, is proposed. The School of Informatics Engineering in Business at the University of Talca, Chile, has a working model with companies with a positive evaluation by students, teachers and employers because it facilitates an early employability of its graduates. The essence of this model has been formalized through a notation for business process modeling (BPMN). In it, all projects are developed in a company by groups of students as part of a course. This formalization has been complemented with the workflow model proposed in this paper. This allows registering and monitoring information associated with the projects being developed, the companies in which they are being executed, the students involved, and the teachers and professionals from the company involved.
\end{abstract}




\section{INTRODUCCIÓN}

Ingeniería en Informática Empresarial (IIE), de la Universidad de Talca-Chile, es una carrera creada en el año 2005, cuyo plan de formación incluye módulos donde los alumnos deben desarrollar proyectos reales en empresas productivas o de servicios. Este plan, recoge elementos propios de la formación profesional dual o educación cooperativa cuya característica central reside en la partición de la formación en dos espacios de aprendizaje: el aula de clase y la empresa (Haddara y Skanes, 2007; Ferns y Zegwaard, 2014). Este modelo de trabajo con empresas (MTE) está consolidado en Alemania, y se considera que ha sido clave en la promoción de la productividad de sus empresas, la integración social y el desarrollo individual (Gohringer, 2002; Gessler, 2009). A ello se agrega la aplicación de la metodología de aprendizaje basado en problemas o proyectos (ABP) o PBL por sus siglas en ingés (Problem or Project Based Learning), en al menos un módulo de cada año de la carrera, en el marco de un plan de formación orientado al desarrollo de competencias (Echavarría, 2010; Fernandez y Duarte, 2013; Díaz-Barriga, 2014). Bajo este esquema, los alumnos se benefician de una formación pertinente a la demanda laboral, mejorando sus posibilidades de empleo junto con el desarrollo de competencias sociales fruto del trabajo teórico-práctico; las empresas se benefician al abrirse la posibilidad de contratar profesionales que ya están imbuidos del ambiente y la cultura empresarial; en tanto que la universidad ve estrechada su vinculación con el medio para la elaboración de programas de formación e investigación pertinentes (Gómez et al., 2015; Jamison et al., 2014; Canales y Schmal, 2013; Marzo et al., 2008; Mesa et al., 2008).

En IIE este MTE ha sido valorado muy positivamente por alumnos, profesores y empleadores, al facilitar una inserción laboral temprana de sus egresados (Schmal, 2012; Nocetti, 2013). Esto fue reconocido nacionalmente al obtener una acreditación por 5 años en el año 2012 (Acredita Cl, 2012). Cabe destacar que más del $95 \%$ de los alumnos matriculados en la carrera provienen de establecimientos educacionales subvencionados, tanto municipales como particulares, lo que no ha sido obstáculo para que el $100 \%$ de sus egresados se encuentren empleados al primer año de egreso (Mifuturo, 2015).

No obstante lo expuesto, en el proceso de acreditación llevado a cabo en el año 2012, se observó la informalidad con que los alumnos desarrollan los proyectos en las empresas, cuyas consecuencias negativas más destacadas son: a) dificultades para gestionar apropiadamente los proyectos; b) disímiles alcances y complejidades entre los distintos proyectos en un mismo módulo; y c) imposibilidad de abordar proyectos de gran tamaño. A ello se agrega la imposibilidad de certificar la experiencia laboral adquirida. Con el fin de subsanar esta informalidad, dentro del plan de mejoramiento comprometido en el proceso de acreditación, se incluyó la necesidad de formalizar el modelo de trabajo con empresas (MTE) que informalmente se estaba llevando a cabo.

De allí que a partir del año 2013 se iniciaron los estudios conducentes a crear un Sistema de Gestión de Proyectos con Empresas (SGPE) con miras a formalizar un modelo de trabajo, lo que se realizó a partir de 2 diagramas: de transición de estados (DTE) y de notación de modelamiento de procesos de negocios (BPMN) (Schmal, 2016)). Bajo este sistema se asume que todo proyecto es desarrollado por grupos de alumnos en una empresa específica y en el marco de un curso. En el año 2014, se pudo observar que no obstante disponerse de un SGPE debidamente formalizado, éste no se implementó apropiadamente ni en toda su extensión. Ello se explica por la libertad que la informalidad proveía a los académicos responsables de los módulos comprometidos con proyectos en empresas, libertad que se veía restringida por la burocratización asociada a la formalización del sistema, en términos de la documentación y plazos exigidos a los distintos actores, en particular, a los profesores.

Lo expuesto llevó, en el año 2015, a revisar la formalización efectuada procurando simplificarlo. Además, se observó la necesidad de automatizarlo mediante un sistema de información basado en workflow como una forma de facilitar el registro y seguimiento de los proyectos desarrollados por los alumnos en las empresas, bajo la supervisión de los profesores. El interés por perseverar en esta senda se debe a que el MTE constituye una de las fortalezas de la carrera más valoradas por los alumnos y egresados. El trabajo con proyectos en empresas reales desde el inicio de la carrera, se ha comprobado que es un factor clave que explica la alta empleabilidad de los egresados y su temprana inserción laboral.

El objetivo de este trabajo es presentar el resultado de este proceso de construcción del SGPE que procura automatizar un MTE orientado: a) en lo operativo, a facilitar el registro de la información asociada a los proyectos que desarrollan los alumnos bajo la supervisión de los profesores; b) en lo táctico, a monitorear el estado en que se encuentran los distintos proyectos; y c) en lo estratégico, a posibilitar la certificación de egresados con experiencia laboral.

La validez del MTE levantado se circunscribe al caso en estudio, esto es, al modelo que se está aplicando en la carrera de IIE de la Universidad de Talca. Por tanto su eventual extensión a otros ámbitos solo es 
posible en la medida que los estados que se definan para los proyectos que desarrollan los estudiantes sean los definidos en este documento, y que los cambios de dichos estados se den bajo las reglas especificadas.

La automatización del MTE persigue facilitar la implementación de un modelo que se ha estado llevando a cabo informalmente, lo que ha dificultado el seguimiento de los proyectos así como la toma de decisiones tanto por parte de los profesores como de la dirección de la carrera. Por tanto, su aporte se centra en la disponibilidad de un sistema de apoyo a la gestión de los proyectos que los estudiantes llevan a cabo en las empresas. Su eventual homologación a otros espacios estará condicionada por las similitudes y/o diferencias de dichos espacios con el caso en estudio.

\section{METODOLOGÍA}

Para el desarrollo del trabajo, en una primera etapa, a través de la técnica de lluvia de ideas (brainstorming), se capturaron los requerimientos de información de los distintos actores (alumnos, profesores, Dirección de Escuela, profesionales y ejecutivos de las empresas). Luego, se modelaron los datos requeridos para satisfacer los requerimientos, los que se representaron a través de un esquema relacional normalizado. Posteriormente, con el apoyo de diagramas (BPMN y SIPOC), se representaron las entradas/salidas de los distintos procesos identificados a través de los diagramas de flujo de datos asociados. Junto con ello se diseñaron las pantallas de entrada para el registro y actualización de la información, además de las pantallas de salida que respondan los requerimientos funcionales (consultas y emisión de reportes). Finalmente, haciendo uso del software Process Maker se procedió a automatizar el MTE ya formalizado.

\section{Modelado de la formalización}

Para modelar el proceso de formalización del MTE se ocuparon diagramas de máquinas de estado de UML 2.0 (Muneton et al., 2007) y de BPMN (Dijkman et al., 2008), una notación gráfica destinada a describir la lógica de los pasos a seguir dentro de un proceso de negocio diseñada para coordinar la secuencia de los procesos y los mensajes que fluyen entre los participantes de las diferentes actividades (Freund et al., 2012). Se trata de un estándar internacional, ya consolidado, para el modelamiento de procesos, gracias a una notación que facilita la comunicación entre profesionales de distintas disciplinas. Posteriormente se ocupó el diagrama SIPOC, herramienta de Six Sigma, para los efectos de identificar las entradas, salidas, los responsables de proveer las entradas y los destinatarios de las salidas (Siddh et al. 2013; Felizzola et al., 2014). Para aplicar BPMN, se ha ocupado el software Bizagi (Pais, 2013), un software de libre disponibilidad, completo y fácil de usar, sin necesidad de disponer de mayores conocimientos informáticos, lo que ayuda a focalizarse en la comprensión de la lógica del proceso en estudio.

\section{Modelado de la automatización}

Para modelar el proceso de automatización del MTE se utilizó el concepto de flujo de trabajo (workflow), que permite el traspaso, total o parcial, de documentos, información o tareas de un participante o usuario a otro para la ejecución de actividades en base a ciertas reglas predefinidas (Van der Alst y Van Hee, 2004). En consecuencia a través de un sistema de workflow es posible asegurar la realización de una secuencia de actividades con la participación de personas y/o sistemas. Por tratarse de un software de uso libre, el nivel de conocimientos existente en torno a la herramienta, así como por su facilidad de uso, se optó por aplicar el software ProcessMaker (Königs, 2012), un software para la administración de workflows que permite diseñar, automatizar y ejecutar flujos de documentos y procesos que ayuda: a) a reducir la documentación física, agilizar y monitorear el seguimiento de los resultados de las actividades que se llevan a cabo; b) a utilizar eficientemente los recursos disponibles; y c) a mejorar los resultados.

\section{MODELO DE TRABAJO EN EMPRESAS (MTE)}

EI MTE se concibió sobre la base de considerar como objeto de estudio el proyecto que los estudiantes desarrollan bajo la supervisión del profesor del módulo en que se desenvuelven, y cuyos resultados (entregables) son validados por un representante de la empresa donde se ejecuta el proyecto. El diseño del MTE implicó definir los posibles estados de cada proyecto, las actividades o eventos que producen los cambios de estado, y la documentación requerida y/o generada por las actividades o eventos definidos. En una primera versión del MTE, se identificaron 10 estados y las actividades que conducen a ellos, como se muestra en la figura 1.

Como consecuencia de las pruebas efectuadas sobre dicha versión, se observó un excesivo grado de centralización en la dirección de la Escuela y la consiguiente sobrecarga de trabajo, lo que conllevaría una pérdida de eficiencia que atentaría contra la adopción del MTE. Fruto de su revisión, se redefinieron las 
actividades que producen los cambios de estados de los proyectos. A continuación se definen las actividades incluidas en el diagrama BPMN de la figura 2.

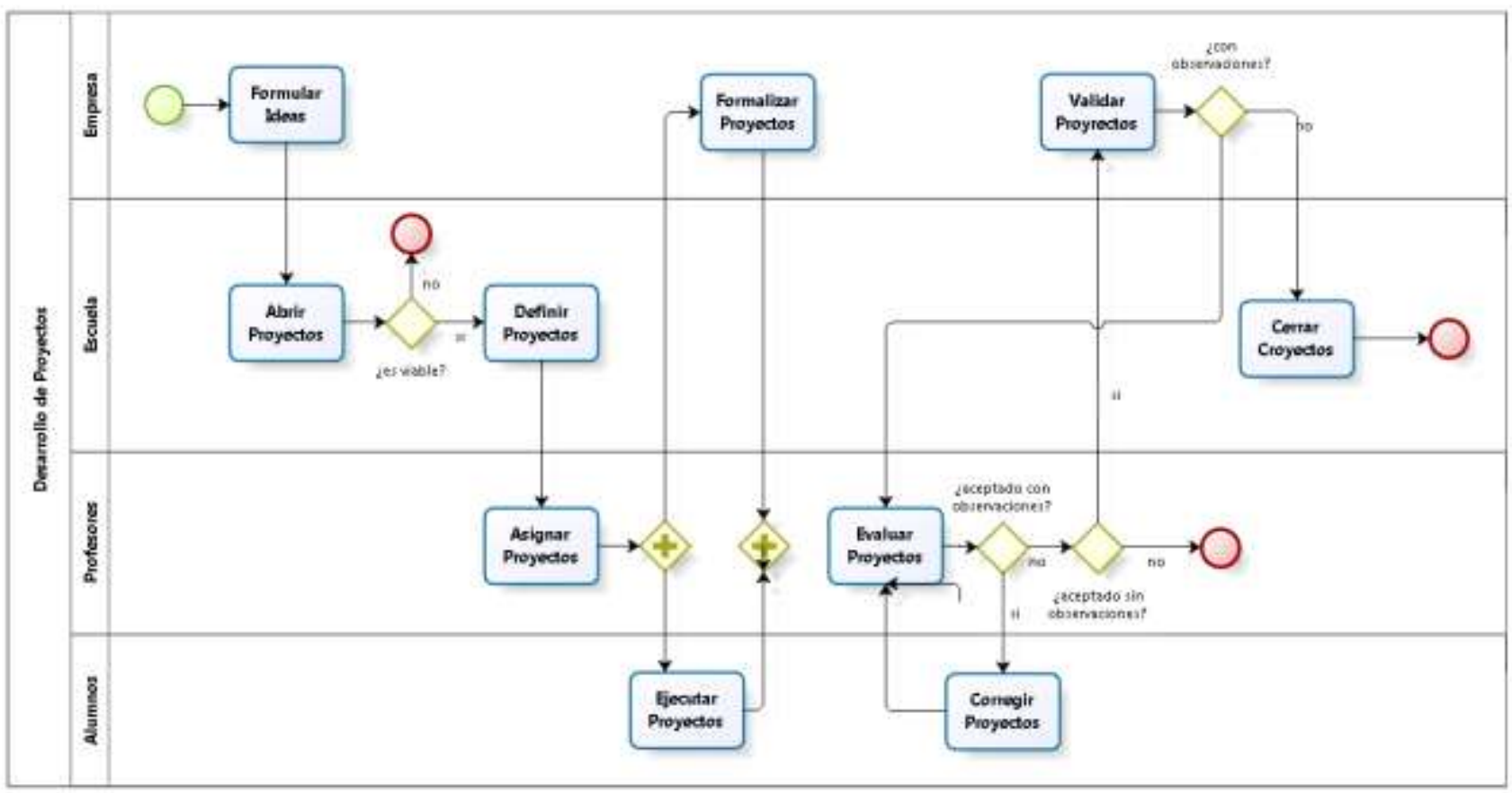

Fig. 1: Proceso de Formalización en BPMN versión inicial

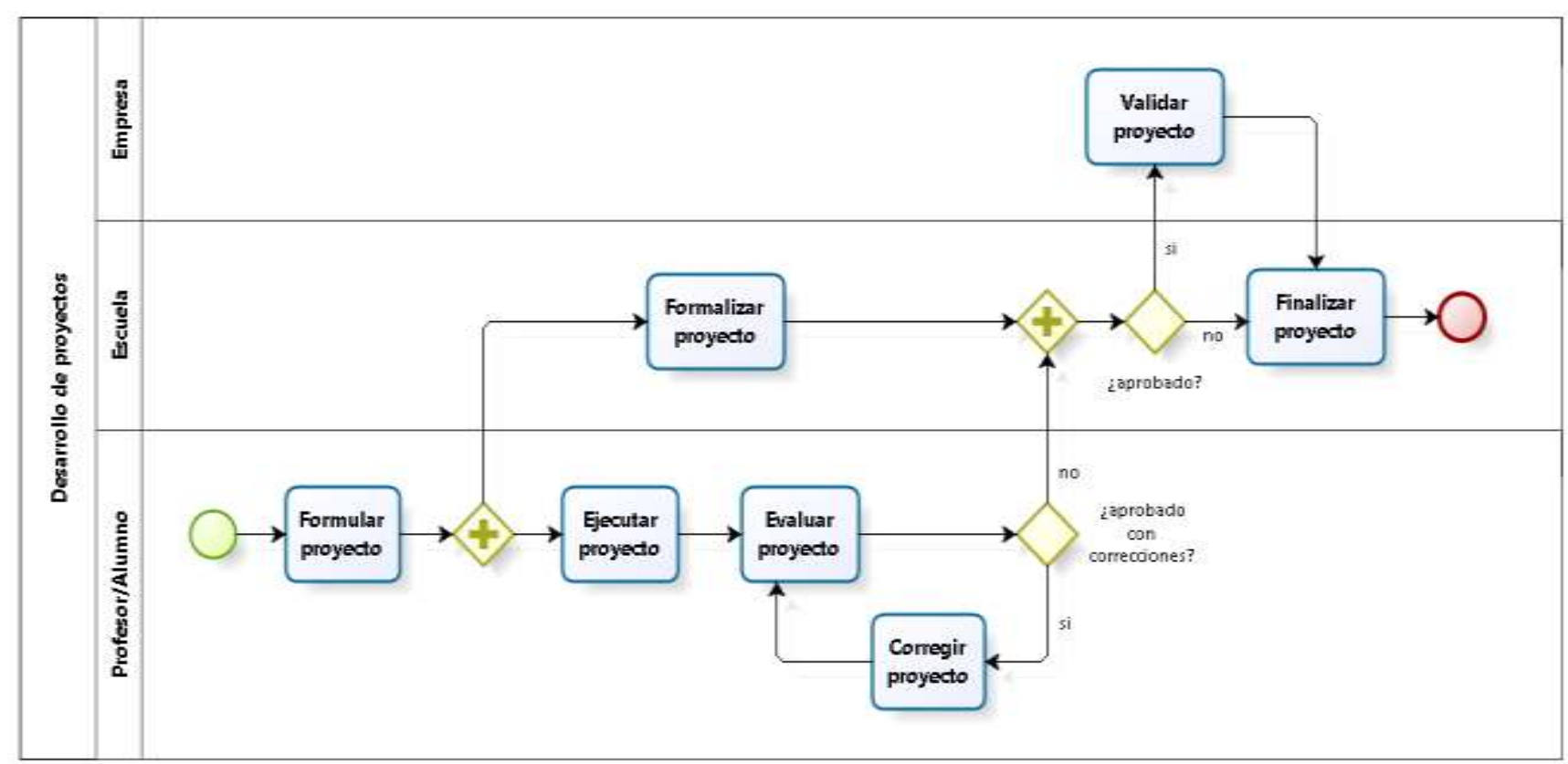

Fig. 2: Proceso de Formalización en BPMN versión final

Formular proyecto: es la actividad donde se describe el proyecto, se delimita su alcance, se identifican sus fecha de inicio y término, los recursos y entregables que compromete, el módulo y la empresa en que se desarrollará el proyecto, además de los alumnos que lo realizarán. Como resultado de esta actividad, el proyecto pasa el estado de formulado.

Formalizar proyecto: es la actividad que protocoliza formalmente los compromisos contraídos por los actores involucrados en el proyecto (empresa, escuela, profesor y alumnos). Completada esta actividad, el proyecto pasa al estado de formalizado.

Ejecutar proyecto: es la actividad desarrollada por los alumnos con miras al cumplimiento de los entregables comprometidos para la empresa de acuerdo a lo acordado tanto con el representante de la empresa, como con el profesor del módulo correspondiente. 
Evaluar proyectos: es la actividad que realizan los profesores responsables de la supervisión de los proyectos. En el caso que un proyecto merezca observaciones, éstas deberán ser informadas a los alumnos para su corrección. Completada esta actividad, el proyecto ha sido aprobado u observado. Esta actividad solo puede realizarse una vez que el proyecto se encuentre formalizado. Solo si el proyecto fue aprobado por el profesor, sus entregables son remitidos a la empresa para su validación antes de su finalización; en caso contrario, si el proyecto fue reprobado por el profesor, el proyecto se da por finalizado.

Corregir proyecto: es la actividad que realizan los alumnos cuyos entregables han sido observados, en cuyo caso los alumnos deben proceder a acoger las observaciones efectuadas por el profesor hasta que éste lo apruebe sin observaciones.

Validar proyectos: es la actividad realizada por el representante de la empresa en que se desarrolla el proyecto, por la cual informa a la Escuela si valida o no el proyecto, señalando su nivel de conformidad con lo realizado.

Finalizar proyectos: es la actividad que realiza el Director de la Escuela para confirmar que ha recibido la validación por parte de la empresa, con la aprobación, o no, de los entregables que fueron comprometidos formalmente.

En el diagrama de la figura 3 se presentan las entradas, salidas y actores o unidades involucradas en las distintas actividades bajo las cuales se ha configurado el MTE.

\begin{tabular}{|c|c|c|c|c|}
\hline S(upplier) & I(nput) & $\mathrm{P}$ (rocess) & O(utput) & C(onsumer) \\
\hline $\begin{array}{c}\text { Alumno } \\
\text { Profesor } \\
\text { Profesional } \\
\text { Empresario }\end{array}$ & $\begin{array}{l}\text { Formulario } \\
\text { presentación } \\
\text { del proyecto }\end{array}$ & $\begin{array}{l}\text { Formular } \\
\text { proyecto }\end{array}$ & $\begin{array}{l}\text { Proyecto } \\
\text { formulado }\end{array}$ & $\begin{array}{l}\text { Escuela } \\
\text { Profesor }\end{array}$ \\
\hline $\begin{array}{l}\text { Escuela } \\
\text { Profesor }\end{array}$ & $\begin{array}{l}\text { Proyecto } \\
\text { formulado }\end{array}$ & $\begin{array}{c}\text { Formalizar } \\
\text { proyecto }\end{array}$ & $\begin{array}{l}\text { Proyecto } \\
\text { formalizado }\end{array}$ & $\begin{array}{c}\text { Escuela } \\
\text { Profesor } \\
\text { Empresa } \\
\text { Alumno }\end{array}$ \\
\hline Escuela & $\begin{array}{l}\text { Proyecto } \\
\text { formulado }\end{array}$ & $\begin{array}{l}\text { Ejecutar } \\
\text { proyecto }\end{array}$ & $\begin{array}{l}\text { Proyecto } \\
\text { ejecutado }\end{array}$ & Profesor \\
\hline Profesor & $\begin{array}{l}\text { Proyecto } \\
\text { ejecutado }\end{array}$ & $\begin{array}{l}\text { Evaluar } \\
\text { proyecto }\end{array}$ & $\begin{array}{l}\text { Proyecto } \\
\text { evaluado }\end{array}$ & $\begin{array}{c}\text { Alumno } \\
\text { Empresa }\end{array}$ \\
\hline Alumno & $\begin{array}{l}\text { Proyecto } \\
\text { evaluado }\end{array}$ & $\begin{array}{l}\text { Corregir } \\
\text { proyecto }\end{array}$ & $\begin{array}{l}\text { Proyecto } \\
\text { corregido }\end{array}$ & Profesor \\
\hline $\begin{array}{l}\text { Alumno } \\
\text { Empresa }\end{array}$ & $\begin{array}{l}\text { Proyecto } \\
\text { evaluado }\end{array}$ & $\begin{array}{l}\text { Validar } \\
\text { proyecto }\end{array}$ & $\begin{array}{l}\text { Proyecto } \\
\text { validado }\end{array}$ & $\begin{array}{l}\text { Escuela } \\
\text { Profesor } \\
\text { Alumno } \\
\end{array}$ \\
\hline Empresa & $\begin{array}{l}\text { Proyecto } \\
\text { validado }\end{array}$ & $\begin{array}{l}\text { Finalizar } \\
\text { proyecto }\end{array}$ & $\begin{array}{l}\text { Proyecto } \\
\text { cerrado }\end{array}$ & $\begin{array}{l}\text { Escuela } \\
\text { Profesor } \\
\text { Alumno }\end{array}$ \\
\hline
\end{tabular}

Fig. 3. Diagrama SIPOC

\section{RESULTADOS}

A continuación se describen las pantallas de entrada asociadas a las actividades identificadas y que comprometen la actuación de los actores que interactúan con el sistema: profesores, la secretaría y la dirección de la Escuela.

Formular proyecto: actividad realizada por el profesor del módulo bajo el cual se llevará a cabo el proyecto, e incluye: a) el registro de los datos generales del proyecto (nombre, descripción y alcance del proyecto; b) el nombre de la empresa, su representante, cargo, teléfono y correo electrónico); c) el detalle de los requerimientos del proyecto (módulo en que se inserta, fecha de inicio y término programado, nivel de complejidad, entregables esperados, actividades y recursos que compromete); e d) la identificación de los alumnos que desarrollarán el proyecto (figura 4).

Formalizar proyecto: actividad realizada por la secretaría de la Escuela que le permitirá descargar e imprimir un documento de protocolo de acuerdo para ser firmado por los alumnos que realizan el proyecto, el profesor que supervisa el proyecto, y el representante de la empresa. Una vez firmado el documento, la secretaría de la Escuela informa al sistema de ello (figura 5). 


\section{FORMULACIÓN DEL PROYECTO}
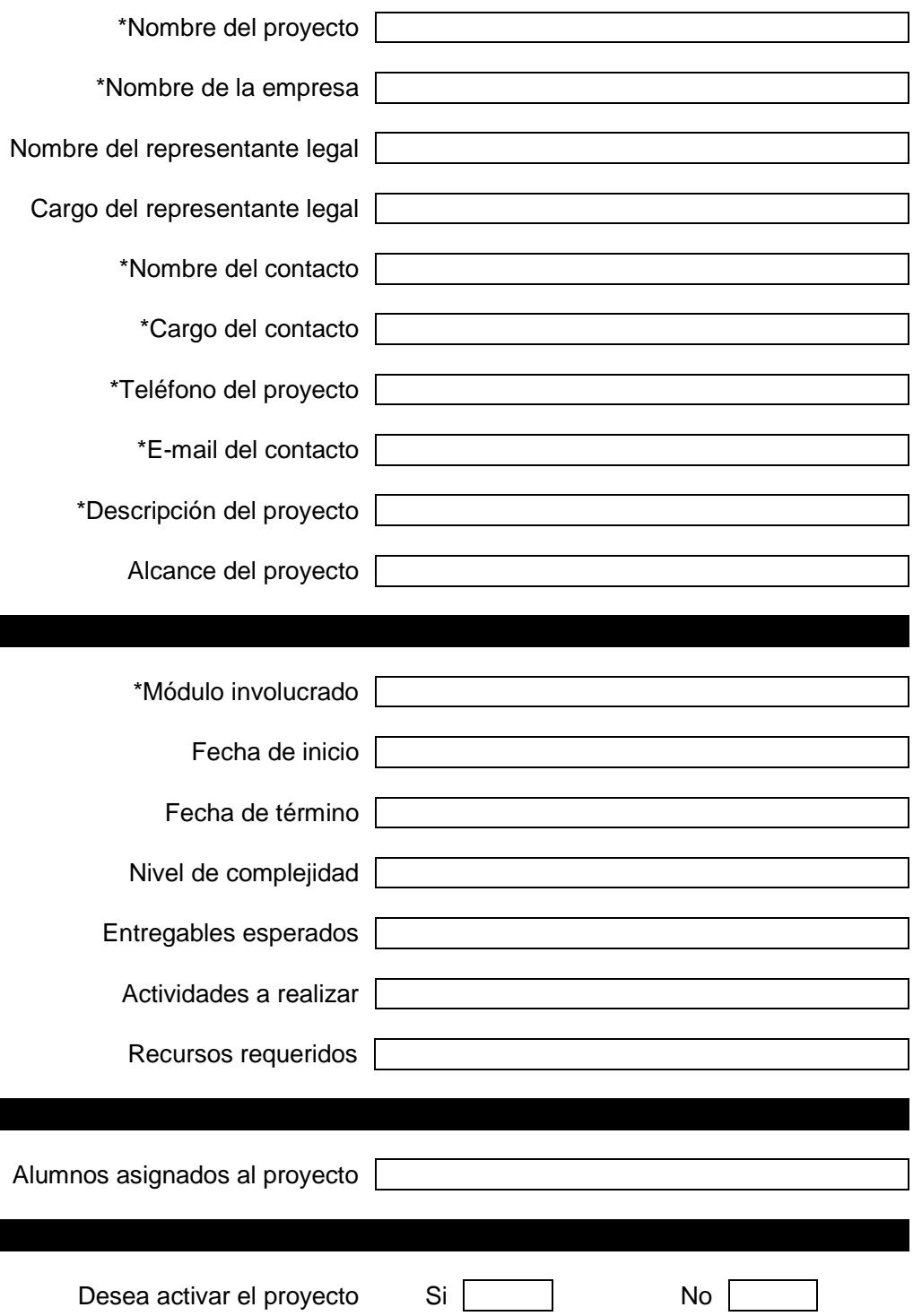

No

Enviar

Fig. 4: Formulario/pantalla de Formulación del Proyecto

Evaluar proyecto: actividad realizada por el profesor del módulo en que se desarrolla el proyecto que permite ingresar la fecha de término del proyecto, la evaluación general (aprobado, aprobado con observaciones, o reprobado) y comentarios generales que merezca al profesor el trabajo realizado por los alumnos. En caso que el proyecto haya sido aprobado o reprobado, la evaluación se da por finalizada; en caso que haya sido aprobado con observaciones que ameriten correcciones de parte del grupo de alumnos, la evaluación no se debe dar por finalizada (figura 6).

Finalizar proyecto: actividad realizada por la dirección de la Escuela que da por cerrado el proyecto, lo que se da cuando: a) la empresa haya entregado su evaluación, ya sea aprobando o reprobando el proyecto; b) el profesor haya reprobado el proyecto (figura 7). Entre las pantallas de salida que contempla el sistema, se incluyen la generación de los siguientes reportes:

Reporte de proyectos a nivel anual: corresponde al listado de los proyectos desarrollados en un año en particular, que incluye nombre del proyecto, módulo y empresa en que se desarrolla, fechas de inicio y término, y su estado; se genera un documento de nombre <año >.doc.

Reporte de proyectos a nivel académico: corresponde al listado de los proyectos supervisados por un profesor en particular, que al igual que el reporte anterior, incluye nombre del proyecto, módulo y empresa 
en que se desarrolla, fechas de inicio y término, y su estado; se genera un documento de nombre $<$ nombreprofesor $>$.doc.

Reporte de proyectos a nivel de alumno: corresponde al listado de proyectos desarrollados por un alumno en particular, que incluye nombre del proyecto, módulo y empresa implicados, fechas de inicio y término, y su estado; se genera un documento de nombre <nombrealumno >.doc

Carta de presentación: corresponde a la carta que identifica al alumno como estudiante de la carrera en la universidad ante la empresa donde se desarrolla el proyecto.

Protocolo de acuerdo: corresponde al acuerdo a ser firmado por las partes (alumnos, profesor, dirección de Escuela y empresa) que oficializa la realización del proyecto en los términos que en él se señalen.

FORMALIZACIÓN DEL PROYECTO

\begin{tabular}{c|l|}
\cline { 2 - 2 } Nombre del proyecto & Rediseño organizacional \\
\cline { 2 - 2 } Nombre de la empresa & Transportes PIPAU \\
\cline { 2 - 2 } Descripción del proyecto & $\begin{array}{l}\text { Levantamiento del modelo del negocio para su } \\
\text { rediseño organizacional si su actual diseño no } \\
\text { está alineado con él. }\end{array}$ \\
\cline { 2 - 2 } \\
Alcance del proyecto & $\begin{array}{l}\text { El rediseño considera el modelo del negocio tal } \\
\text { cual es, asumiendo que no será modificado. }\end{array}$ \\
\cline { 2 - 2 }
\end{tabular}

\begin{tabular}{rl|} 
Módulo involucrado & Rediseño Organizacional \\
Fecha de inicio & 1 sep 2015 \\
Fecha de término & 15 dic 2015 \\
Nivel de complejidad & mediano \\
Entregables esperados & $\begin{array}{l}\text { Informe ejecutivo } \\
\text { Informe final } \\
\text { Presentación oral } \\
\text { Video }\end{array}$ \\
\hline
\end{tabular}

Actividades que compromete el proyecto Levantar el modelo del negocio a partir de la misión, visión y estrategia de la empresa Levantar el diseño organizacional que posee la empresa

Analizar el diseño organizacional de la empresa en base a los modelos organizacionales existentes

Proponer un rediseño organizacional debidamente sustentado.

Recursos requeridos por el proyecto 2 días de trabajo de los ejecutivos de la empresa $\$ 150,000$ para transporte y alimentación de los alumnos durante las jornadas de trabajo en la empresa.

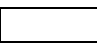

No

Enviar

Fig. 5: Formulario/pantalla de Formalización del Proyecto 


\section{EVALUACIÓN DEL PROYECTO}

Identificación del Proyecto

Nombre del proyecto Rediseño organizacional de una empresa de transportes

Nombre de la empresa Transportes PIPAU

Módulo asignado Rediseño Organizacional

Profesor supervisor Rodolfo Schmal

Alumnos asignados Carlos Eusebio Figueroa; Eduardo Felipe Canales

Entregables del proyecto Informe ejecutivo; Informe final; Presentación oral; Video

Detalle de la Evaluación del Proyecto

Fecha de término 15 dic 2015

Evaluación General Aprobado Reprobado Observado

Comentarios generales

Valor estimado del Proyecto (\$)

\section{Evaluación del Proyecto}

Confirma que la evaluación está terminada

Si

No

Enviar

Fig. 6: Formulario/pantalla de Evaluación del Proyecto

FINALIZACIÓN DEL PROYECTO

\section{Identificación del Proyecto}

Nombre del proyecto Rediseño organizacional de una empresa de transportes

Nombre de la empresa Transportes PIPAU

Módulo asignado Rediseño Organizacional

Profesor supervisor Rodolfo Schmal

Alumnos asignados Carlos Eusebio Figueroa; Eduardo Felipe Canales

\section{Evaluación General del Proyecto}

Evaluación del profesor Aprobado

Evaluación General de la Empresa

Aceptado

Rechazado

Comentarios generales

Valor estimado del Proyecto (\$)

\section{Cierre del Proyecto}

Confirma el cierre del proyecto

Si

No

Enviar

Fig. 7: Formulario/pantalla de Finalización del Proyecto 


\section{DISCUSIÓN}

El sistema de automatización surge a 10 años del inicio de la carrera de IIE y de la aplicación de un modelo de aprendizaje basado en el principio de "aprender haciendo" (learning by doing). Este modelo de aprendizaje ha logrado sostenerse en el tiempo y se ha constituido en la principal fortaleza de la carrera, reconocida en el proceso de acreditación al que se sometió la carrera en el año 2012 luego de un proceso de autoevaluación efectuado el año anterior (2011). Tanto el proceso de acreditación externa, como el de autoevaluación interna, identificaron como una de sus debilidades, la informalidad con que es implementado este modelo, lo que atenta contra su consolidación y, junto con ello, arriesga la pérdida de lo que actualmente se percibe como una fortaleza.

En este plano surge la necesidad de sistematizar lo que se hace, la forma de trabajar con las empresas, en una forma orgánica, que empareje la cancha a los distintos actores, estableciendo reglas de juego claras y conocidas, que reduzcan la discrecionalidad y provean mayores niveles de seguridad tanto a profesores, como alumnos y empresas en las que se realizan los proyectos. Por tanto, el primer paso realizado consistió en la formalización del MTE, el que una vez socializado entre el cuerpo de profesores, abre espacio a su automatización de modo que se facilite su implementación, puesto que en caso contrario se corre el riesgo de que la informalidad permanezca en el tiempo. De hecho así ha sido, como lo prueba que no obstante que se viene insistiendo en la necesidad de formalizar el MTE, hasta la fecha esta formalización no se ha hecho carne. En consecuencia, la automatización del MTE propuesta apunta a forzar su implementación, lo que se está dando en el presente año mediante su prueba en dos de los módulos de la carrera en los que se trabaja en base a proyectos en empresas.

A partir del año 2016 se aspira que este sistema de automatización se encuentre implementado en la totalidad de los módulos que operan con proyectos en empresas. Cabe destacar que para el éxito del sistema es imprescindible su apropiada socialización entre el cuerpo de profesores y considerar las observaciones que les merezca. Sin la voluntad de los profesores se arriesga la pérdida del esfuerzo desplegado y del propio MTE.

\section{CONCLUSIONES}

En torno al sistema de automatización del MTE, se concluye que debe facilitar: 1) la implementación de la formalización; 2) su uso dado que es fruto de sucesivas pruebas en el marco de 2 módulos de la carrera que han dado origen a cambios orientados a su simplificación; 3) la gestión de los proyectos por parte de los profesores y la dirección de la Escuela; 4) un mejoramiento en la calidad de los proyectos que se llevan a cabo; y 5) la certificación de los proyectos desarrollados y con ello demostrar que una vez egresados, lo hacen con experiencia laboral.

\section{REFERENCIAS}

Acredita Cl; Acuerdo de Acreditación N²13, Agencia Acreditadora del Colegio de Ingenieros, 9 p. (2012) Disponible en https://www.cnachile.cl/res/preg/RES-PREG-01126-01.pdf Acceso: Junio 24, (2015)

Canales, T. y Schmal, R.; Trabajando con Pósteres: una herramienta para el desarrollo de habilidades de comunicación en la educación de pregrado, Formación Universitaria, Vol 6 № 1, pp.41-52 (2013)

Diaz-Barriga, A.; Construcción de programas de estudio en la perspectiva del enfoque de desarrollo de competencias, Perfiles Educativos, Vol. 36 №143, pp. 142-162 (2014)

Dijkman, R.M., Dumas, M., Ouyang, C.; Semantics and analysis of business process models in BPMN, Information and Software Technology, Vol. 50 №12, pp. 1281-1294 (2008).

Echavarría, M.V.; Problem-based learning application in engineering, Revista EIA (Escuela de Ingeniería de Antioquia, Medellín (Colombia), Vol 14 № 1, pp. 85-95 (2010)

Felizzola, H. y Luna, C.; Lean Six Sigma en pequeñas y medianas empresas: un enfoque metodológico, Revista Ingeniare, Vol. 22 № 2, pp.263-277 (2014)

Fernández, F. y Duarte, J.; El Aprendizaje basado en Problemas como Estrategia para el Desarrollo de Competencias Específicas en Estudiantes de Ingeniería, Formación Universitaria, Vol. 6 № 5, pp. 29-38 (2013) 
Ferns, S. y Zegwaard K.E.; Critical assessment issues in work-integrated learning, Asia-Pacific Journal of Cooperative Education, Special Issue, Vol. 15 № 3, pp. 1879-188 (2014)

Freund, J. Rucker, B. y Hitpass, B.; BPMN 2.0 Manual de Referencia y Guía Práctica, Santiago de Chile, $2^{2}$ edición, Depto. Informática- Universidad Técnica Federico Santa María, 283 pp. (2012)

Gessler, M.; La formación profesional en Alemania. En Gairín, J., Essombra, M. A. y Muntané, D. (Coords.). La calidad de la Formación Profesional en Europa hoy. Madrid: Wolters Kluwer Educación, 352 pp. (2009)

Gómez, M.C., Manrique, B. y Gasca G.P.; Propuesta de evaluación de habilidades blandas en Ingeniería de Software por medio de proyectos Universidad-Empresa, Revista Educación en Ingeniería, Vol $10 \mathrm{~N}^{\circ}$ 19, pp. 131-140 (2015)

Gohringer, A.; University of Cooperation Education - Karlsruhe: The Dual System of Higher Education in Germany. Asia-Pacific Journal of Cooperative Education, Vol. 3 № 2, pp. 53-58 (2002)

Haddara, M. y Skanes, H.; A reflection cooperative education: from experience to experiential learning, AsiaPacific Journal of Cooperative Education, Vol. $8 \mathrm{~N}^{\circ}$ 1, pp. 67-76 (2007)

Jamison A., Kolmos, A. y Holgaard, J.E.; Hybrid Learning: An Integrative Approach to Engineering Education, Vol. $103 \mathrm{~N}^{\circ} 2$, pp.253-273 (2014)

Königs, O.; Open Source and Workflow im Unternahmen. Ed. Vrlag GmbH, Hamburgo-Alemania, pp. 140 (2012)

Marzo, M., Pedraja, M. y Rivera, P.; Un modelo de relaciones empresa-universidad, Revista Europea de Dirección y Economía de la Empresa, Vol 17 № 1, pp. 39-56 (2008)

Mesa, J.M., Álvarez, J.V., Villanueva, J.M. y De Cos, F.J.; Actualización de Métodos de EnseñanzaAprendizaje en Asignaturas de Dirección de Proyectos de Ingeniería, Formación Universitaria, Vol. $1 \mathrm{~N}^{\circ} 4$, pp. 23-28 (2008)

Mifuturo; Buscador de Empleabilidad e Ingresos. (en línea: http://mifuturo.cl/index.php/futurolaboral/buscador-por-carrera-d-institucion, acceso: 10 de Noviembre 2015)

Montoya, F.J. y Aguilar, J.; La Relación Universidad-Empresa en las Prácticas Empresariales: Un Modelo Conceptual desde las Técnicas de Generación de Ideas, Journal of Technology Management \& Innovation, Vol. 8 suppl. 1, pp. 196-208 (2013)

Muneton, A. Zapata, C. y Arango, F.; Reglas para la generación automática de código definidas sobre metamodelos simplificados de los diagramas de clases, secuencias y máquinas de estado de UML 2.0. Dyna. Vol. 74 № 153, pp. 267-283 (2007)

Nocetti, V.; Rompiendo los paradigmas en la educación y formación profesional de ingenieros: la experiencia de consultorías en Ingeniería Informática Empresarial de la Universidad de Talca. XXVI Congreso Chileno de Educación en Ingeniería, Viña del Mar-Chile (2013)

Pais, J.R.; BPM (Business Process Management). Ed. Pedro Robledo, Madrid-España, pp. 300 (2013)

Schmal, R.; Un nuevo profesional para la gestión organizacional, Revista del Instituto Internacional de Costos, Edición Especial XII Congreso Internacional de Costos, pp. 87-89. (2012)

Schmal, R.; Rivero, S. y Vidal, C.; Formalización de un modelo de trabajo con empresas en una carrera de ingeniería. Ingeniare, Vol. $24 \mathrm{~N}^{\circ}$ 1, pp. 149-157 (2016)

Siddh, M.M., Gadekar, G., Soni, G. y Jain, R.; Lean Six Sigma Approach for Quality and Business Performance, Global Journal of Management and Business Studies, Vol. 3 Nº 6, pp. 589-594 (2013)

Van der Aalst, W y Van Hee, K.; Workflow Management: Models, Methods, and Systems. Ed. MIT Press, Cambridge-USA, pp. 369 (2004) 